\title{
Association between modified simple protein-energy wasting (PEW) score and all- cause mortality in patients receiving maintenance hemodialysis
}

Shunsuke Yamada ${ }^{1}$, Toshiaki Nakano ${ }^{1 *}$ (D), Shoji Tsuneyoshi ${ }^{1}$, Hokuto Arase ${ }^{1}$, Sho Shimamoto ${ }^{1}$, Masatomo Taniguchi ${ }^{2}$, Masanori Tokumoto ${ }^{3}$, Hideki Hirakata ${ }^{2}$, Hiroaki Ooboshi ${ }^{3}$, Kazuhiko Tsuruya ${ }^{4}$ and Takanari Kitazono ${ }^{1}$

\begin{abstract}
Background: Protein-energy wasting (PEW) is a frequently observed complication that leads to increased mortality in hemodialysis patients. However, a multifaceted assessment of PEW by combined objective nutritional parameters has not yet been established.

Methods: In total, 144 Japanese patients receiving maintenance hemodialysis at a hemodialysis center were retrospectively followed for 7 years. The primary outcome was all-cause death. The main exposure was a modified simple PEW score $(0,1,2,3$, or 4), calculated from four parameters: serum albumin and creatinine levels, normalized protein catabolic rate, and body mass index. These parameters are included in the subcategories of PEW as defined by the International Society of Renal Nutrition and Management. The cutoff values of the modified simple PEW score components were based on the receiver operating characteristics curves determined by univariate logistic regression analyses. Risk estimates for all-cause mortality were calculated by the Cox proportional hazards model adjusted for potential confounding factors.
\end{abstract}

Results: During the median 5.7-years follow-up period, 37 patients died of any cause. When patients were divided into three subgroups (G1-G3) based on the modified simple PEW score, a multivariable-adjusted analysis showed that the risks of all-cause death in groups G2 and G3 were significantly higher than in the lowest score group (G1), with hazard risk (95\% confidence interval) $3.10(1.16-8.26)(P=0.024)$ and $5.68(1.85-17.45)(P=0.002)$, respectively.

Conclusions: The modified simple PEW score is a useful composite indicator of nutritional status that stratifies the risk of all-cause mortality in patients undergoing maintenance hemodialysis.

Keywords: Albumin, Body mass index, Creatinine, Hemodialysis, Normalized protein catabolic rate, Protein energy wasting

\footnotetext{
*Correspondence: toshink@med.kyushu-u.ac.jp

'Department of Medicine and Clinical Science, Graduate School of Medical

Sciences, Kyushu University, 3-1-1 Maidashi, Higashi-Ku, Fukuoka 8128582,

Japan

Full list of author information is available at the end of the article
}

(C) The Author(s). 2020 Open Access This article is licensed under a Creative Commons Attribution 4.0 International License, which permits use, sharing, adaptation, distribution and reproduction in any medium or format, as long as you give appropriate credit to the original author(s) and the source, provide a link to the Creative Commons licence, and indicate if changes were made. The images or other third party material in this article are included in the article's Creative Commons licence, unless indicated otherwise in a credit line to the material. If material is not included in the article's Creative Commons licence and your intended use is not permitted by statutory regulation or exceeds the permitted use, you will need to obtain permission directly from the copyright holder. To view a copy of this licence, visit http://creativecommons.org/licenses/by/4.0/ The Creative Commons Public Domain Dedication waiver (http://creativecommons.org/publicdomain/zero/1.0/) applies to the data made available in this article, unless otherwise stated in a credit line to the data. 


\section{Introduction}

Malnutrition or undernutrition is common in patients receiving hemodialysis [1]. The underlying causes for malnutrition in these patients are attributed to increased catabolism, decreased appetite and food intake, nutrient loss through the hemodialyzer, and dietary protein restriction for phosphate control [2]. These patients are at increased risk for morbidity and mortality [3]. To date, assessment of nutritional status in combination with effective intervention to treat malnutrition or undernutrition has been a challenging theme in this population.

Protein-energy wasting (PEW) is a term that was proposed by a panel of the International Society of Renal Nutrition and Management (ISRNM) in 2009 [4]. The ISRNM has defined PEW as a state of decreased body stores of protein and energy fuels (body protein and fat mass). The ISRNM also proposed diagnostic criteria for PEW with four distinct categories: (i) biochemical indicators such as serum albumin or prealbumin; (ii) low body weight, reduced fat, or weight loss; (iii) decreased muscle mass; and (iv) low protein or energy intake. In the clinical setting, it would be convenient to integrate all the PEW criteria into one index or risk score and use this integrated score for the periodic assessment of PEW in patients with chronic kidney disease. Recently, Moreau-Gaudry et al. reported a simple PEW score using four nutritional parameters corresponding to four categories of PEW and integrating subscores into a single value that enables semiquantitative assessment of PEW in patients undergoing hemodialysis [5]. Serum levels of albumin and creatinine $(\mathrm{Cr})$, normalized protein catabolic rate (nPCR), and body mass index (BMI) were used for the score calculation. These nutritional parameters are established nutritional markers and reported to be associated with mortality in patients receiving hemodialysis [6-9]. Lopes et al. showed that the simple PEW score predicted all-cause mortality in European patients undergoing hemodialysis. However, racial, habitual, and social backgrounds make for substantial differences in the nutritional status and parameters of this patient population across countries [10]. Therefore, it is reasonable to speculate that some of the cutoff values for PEW categories should account for those differences.

The aim of the present study was threefold. The first aim was to create a modified simple PEW score (mPEWS) for Japanese patients undergoing hemodialysis by adjusting the original simple PEW score created by Moreau-Gaudry A et al. by changing some of the cutoff values of PEW components. The second aim was to elucidate whether the mPEW-S accurately predicts all-cause mortality in Japanese patients receiving hemodialysis. The third aim was to show that some of the cutoff values of the PEW components should be determined depending on the targeting hemodialysis population. For these aims, we retrospectively recruited patients receiving maintenance hemodialysis at a single hemodialysis center in Japan. In this study, we used the geriatric nutritional risk index (GNRI) and $\mathrm{Cr}$ index as pre-established nutritional indexes and references in patients receiving hemodialysis $[11,12]$.

\section{Patients and methods}

\section{Study design and participants}

This was a single-center, retrospective, observational study consisting of 144 outpatients with end-stage kidney disease who had been receiving maintenance hemodialysis therapy at the Fukuoka Renal Clinic on December 1, 2011. Patients were followed until death, transfer to another hospital, or loss to follow-up. Patients who were lost to follow-up during the observation period were regarded as "censored" on the day of the final hospital visit and were also included in the analyses. The study was performed in accordance with the principle of the Declaration of Helsinki. The study protocol was reviewed and approved by the institutional review board at Fukuoka Dental College (2012-256). Written informed consent was obtained from each patient prior to study participation.

\section{Primary outcomes and main exposure}

The primary outcome was death by any-cause. The main exposure was the mPEW-S. The original simple PEW score proposed by Moreau-Gaudry et al. used serum albumin as serum biochemistry, BMI as body weight, serum $\mathrm{Cr}$ /body surface area (BSA) as skeletal muscle mass, and nPCR as protein intake [5]. BSA was calculated by the following formula: BSA $\left(\mathrm{m}^{2}\right)=$ $0.007184 \times$ (height in centimeter $)^{0.725} \times$ (body weight in kilogram $)^{0.425}$. The original cutoff values for each PEW category by Moreau-Gaudry A et al. were $3.8 \mathrm{~g} / \mathrm{dL}, 23$ $\mathrm{kg} / \mathrm{m}^{2}, 380 \mu \mathrm{mol} / \mathrm{L} / \mathrm{m}^{2}$, and $0.8 \mathrm{~g} / \mathrm{kg} /$ day, respectively. The cut-of values set for serum albumin level $(3.8 \mathrm{~g} / \mathrm{dl})$ and $\mathrm{nPCR}(0.8 \mathrm{~g} / \mathrm{kg} /$ day $)$ were derived from the cutoff values set by the ISRNM [4]. As for cutoff values for serum Cr/BSA and BMI, Moreau-Gaudry A et al. determined those cutoff values based on the distribution of serum $\mathrm{Cr} / \mathrm{BSA}$ and BMI among their study population [5]. However, $380 \mu \mathrm{mol} / \mathrm{L} / \mathrm{m}^{2}$ for serum $\mathrm{Cr} / \mathrm{BSA}$ was too low and $23 \mathrm{~kg} / \mathrm{m}^{2}$ for BMI appeared to be too high for the Japanese population. The cutoff values were calculated by the receiver operation characteristic (ROC) curves for each nutritional surrogate by using non-adjusted logistic regression analysis for all-cause mortality and then rounded considering the distribution of those values based on the current study and database of Japanese hemodialysis patients provided by The Japanese Society for Dialysis Therapy (https://www.jsdt.or.jp/Overview_2.html). The unit of serum $\mathrm{Cr}$ was converted from $\mathrm{mg} / \mathrm{dL}$ to $\mu \mathrm{mol} / \mathrm{L}$ by the 
following formula: serum $\mathrm{Cr}(\mu \mathrm{mol} / \mathrm{L})=$ serum $\mathrm{Cr}(\mathrm{mg} / \mathrm{dL})$ $\times 10000$ / 113 (molecular weight for $\mathrm{Cr}$ ).

\section{Data collection}

Demographic data, including dialysis-related parameters, were retrospectively collected by reviewing medical charts. Biochemical parameters used in the present analyses were collected at the start of the dialysis session following the longest interdialytic period (2 days) on the first week of December 2011 and December 2012. Serum calcium levels were corrected using Payne's formula only if patients had a serum albumin level $<4.0 \mathrm{~g} / \mathrm{dL}$ [13]. Serum parathyroid hormone (PTH) levels were determined by immunoradiometric assay as whole PTH and were reported as intact $\mathrm{PTH}$ using the following formula: serum intact PTH $(\mathrm{pg} / \mathrm{mL})=1.7 \times$ whole PTH (pg/mL) [14]. Additional data collected included levels of blood hemoglobin, urea nitrogen, and calcium, as well as serum $\mathrm{C}$-reactive protein.

\section{Calculation of pre-established nutritional indexes}

GNRI and modified $\mathrm{Cr}$ index were calculated as previously reported $[11,12]$ :

GNRI $=14.89 \times$ serum albumin $(\mathrm{g} / \mathrm{dL})+41.7 \times$ body weight $(\mathrm{kg}) /$ ideal body weight

Modified $\mathrm{Cr}$ index $(\mathrm{mg} / \mathrm{kg} /$ day $)=16.21+1.12 \times(1$ if male, 0 if female)

$-0.06 \times$ age (years)

$-0.08 \times$ single-pooled $\mathrm{Kt} / \mathrm{V}^{*}$ for urea

$+0.009 \times$ serum $\mathrm{Cr}$ level before dialysis $(\mu \mathrm{mol} / \mathrm{L})$.

* The Kt/V ratio represents the plasma volume (V) cleared of urea $(\mathrm{Kt})$ during hemodialysis relative to the distribution volume of urea.

Body weight/ideal body weight was set to 1 when the body weight exceeded the ideal body weight. Ideal body weight was calculated as $22 \times$ height (meters) $\times$ height (meters).

\section{Statistical analysis}

Normally distributed continuous variables, non-normally distributed continuous variables, and categorical data were described as mean (standard deviation), median (interquartile range), and percentage, respectively, unless otherwise specified.

The distribution of baseline characteristics in subgroups based on the mPEW-S (G1, G2, and G3) was compared using the following trend analyses: the Cochran-Armitage test was used for categorical variables, and the JonckheereTerpstra test was used for continuous variables. To determine correlation among the mPEW-S, the original simple PEW score, GNRI, or modified Cr index, Spearman's rank-order correlation was used. Unadjusted, age- and sex-adjusted, and multivariable-adjusted hazard ratios (HRs) and 95\% confidence intervals (CIs) for all-cause mortality were estimated using the Cox proportional hazards risk models. The multivariable-adjusted model included the following covariates: age, sex, presence of diabetic nephropathy, history of cardiovascular events, hemodialysis history, serum levels of calcium, phosphate, and PTH. To compare the predictability performance for all-cause mortality between the original simple PEW score and the mPEW-S, we calculated cstatistics by ROC, net reclassification improvement (NRI), and integrated discrimination improvement (IDI).

A two-tailed $P$ value $<0.05$ was considered statistically significant in all analyses. All statistical analyses were performed using JMP Pro 14.2.0 for Windows (SAS Institute, Inc., Tokyo, Japan) and R 3.5.1 (http://cran.rproject.org).

\section{Results}

Determination of cutoff values for each PEW parameter

In total, 144 Japanese patients undergoing maintenance hemodialysis were included in the present analysis. To determine the cutoff values for each PEW parameter appropriate for Japanese hemodialysis patients, we first conducted univariate logistic regression analyses by setting all-cause death as an endpoint and determined ROC curves. The cutoff values for serum albumin, BMI, serum $\mathrm{Cr} / \mathrm{BSA}$, and nPCR were $3.80 \mathrm{~g} / \mathrm{dL}, 18.72 \mathrm{~kg} / \mathrm{m}^{2}, 506.7$ $\mu \mathrm{mol} / \mathrm{L} / \mathrm{m}^{2}$, and $0.789 \mathrm{~g} / \mathrm{kg} /$ day, respectively. Then, after considering the distribution of the four parameters of PEW in the hemodialysis patients registered in the database of the Japanese Society for Dialysis Therapy ((https:// www.jsdt.or.jp/Overview_2.html) and the original cutoff values of the ISRNM, we rounded the cutoff values of BMI, serum Cr Cr/BSA, and nPCR and ultimately set the cutoff values for the four categories of PEW as $3.8 \mathrm{~g} / \mathrm{dL}$ for serum albumin, $18.5 \mathrm{~kg} / \mathrm{m}^{2}$ for BMI, $500 \mu \mathrm{mol} / \mathrm{L} / \mathrm{m}^{2}$ for serum $\mathrm{Cr} / \mathrm{BSA}$, and $0.8 \mathrm{~g} / \mathrm{kg} /$ day for $\mathrm{nPCR}$. For the cutoff value of serum $\mathrm{Cr} / \mathrm{BSA}, 500 \mu \mathrm{mol} / \mathrm{L} / \mathrm{m}^{2}=5.65 \mathrm{mg} /$ $\mathrm{dL} / \mathrm{m}^{2}$; therefore, if a patient's BSA was $1.6 \mathrm{~m}^{2}$, the patient's serum $\mathrm{Cr}$ was almost $9 \mathrm{mg} / \mathrm{dL}$.

Based on the above cutoff values, modified scoring system for PEW was created and is shown in Table 1. The lowest value was 0 and the highest value was 4 . Patients with a higher $\mathrm{MPEW}-\mathrm{S}$ were supposed to be more malnourished and have a higher risk of morbidity and mortality. Because the numbers of patients in mPEW-S 1,3 , and 4 were relatively small compared with those in mPEW-S 0 and 2, to further elucidate the association between $\mathrm{mPEW}-\mathrm{S}$ and mortality, patients were divided into subgroups based on the mPEW-S; G1, mPEW-S 0 or 1; G2, mPEW-S 2; and G3, mPEW-S 3 or 4.

\section{Patients' characteristics}

From the cutoff values of the original simple PEW score and $\mathrm{mPEW}-\mathrm{S}$, the number and proportion of patients in each subcategory is shown in Fig. 1. 
Table 1 Scoring system of the modified simple PEW score

\begin{tabular}{ll}
\hline Variable & Score \\
\hline Serum albumin $(\mathrm{g} / \mathrm{dL})$ & \\
$\geq 3.8$ & 0 \\
$\quad<3.8$ & 1 \\
Serum Cr/BSA $\left(\mu \mathrm{mol} / \mathrm{L}^{\mathrm{m}} \mathrm{m}^{2}\right)$ & \\
$\quad \geq 500$ & 0 \\
$<500$ & 1 \\
$\mathrm{BMI}\left(\mathrm{kg} / \mathrm{m}^{2}\right)$ & \\
$\geq 18.5$ & 0 \\
$<18.5$ & 1 \\
$\mathrm{nPCR}(\mathrm{g} / \mathrm{kg} /$ day $)$ & \\
$>0.8$ & 0 \\
$<0.8$ & 1 \\
Total score & $0-4$
\end{tabular}

Total modified PEW score is the simple sum of each value of four subcategories of PEW

$B M I$ body mass index, $B S A$ body surface area, $C r$ creatinine, $n P C R$ normalized protein catabolic rate, $P E W$ protein-energy wasting

The scoring system for the mPEW-S is shown in Table 1 . Fifty patients showed score 0,10 patients score 1, 63 patients score 2, 18 patients score 3 , and 3 patients score 4. Patients were then classified into one of three groups (G1-G3) according to the mPEW-S: G1, G2, and G3. Namely, a total of 60 patients were classified as G1 (mPEW-S 0 or 1), 63 patients were G2 (mPEW-S 2 ), and 21 patients were G3 (mPEW-S 3 or 4).
Table 2 shows the baseline characteristics in each subgroup divided by the mPEW-S. Patients with a higher mPEW-S were significantly $(P<0.05)$ older and showed lower $\mathrm{nPCR}$, body weight, and BMI, blood hemoglobin, serum albumin, urea nitrogen, $\mathrm{Cr}$, and calcium as well as a higher serum C-reactive protein. There was no tendency regarding the association between $\mathrm{mPEW}-\mathrm{S}$ and medications.

\section{Correlation between mPEW-S and two nutritional indexes} To determine the validity of our newly developed mPEW-S as a nutritional score, we examined whether the mPEW-S showed an association with the modified $\mathrm{Cr}$ index and GNRI, established scores for good nutrition, by using the mPEW-S as a continuous variable. As shown in Fig. 2a, b, a higher mPEW-S was significantly $(P<0.05)$ and negatively correlated with a lower $\mathrm{Cr}$ index and GNRI; the coefficients of determination were 0.29 and 0.54 , respectively.

We also examined the correlation between the original simple PEW score and those two nutritional indexes by using the $\mathrm{MPEW}-\mathrm{S}$ as a continuous variable. As shown in Fig. 2c, d, the original PEW score was significantly $(P$ $<0.05$ ) and negatively correlated with $\mathrm{Cr}$ index and GNRI and the coefficients of determination were 0.25 and 0.39 , respectively. When coefficients of determination were compared across those PEW scores, the values were almost comparable.

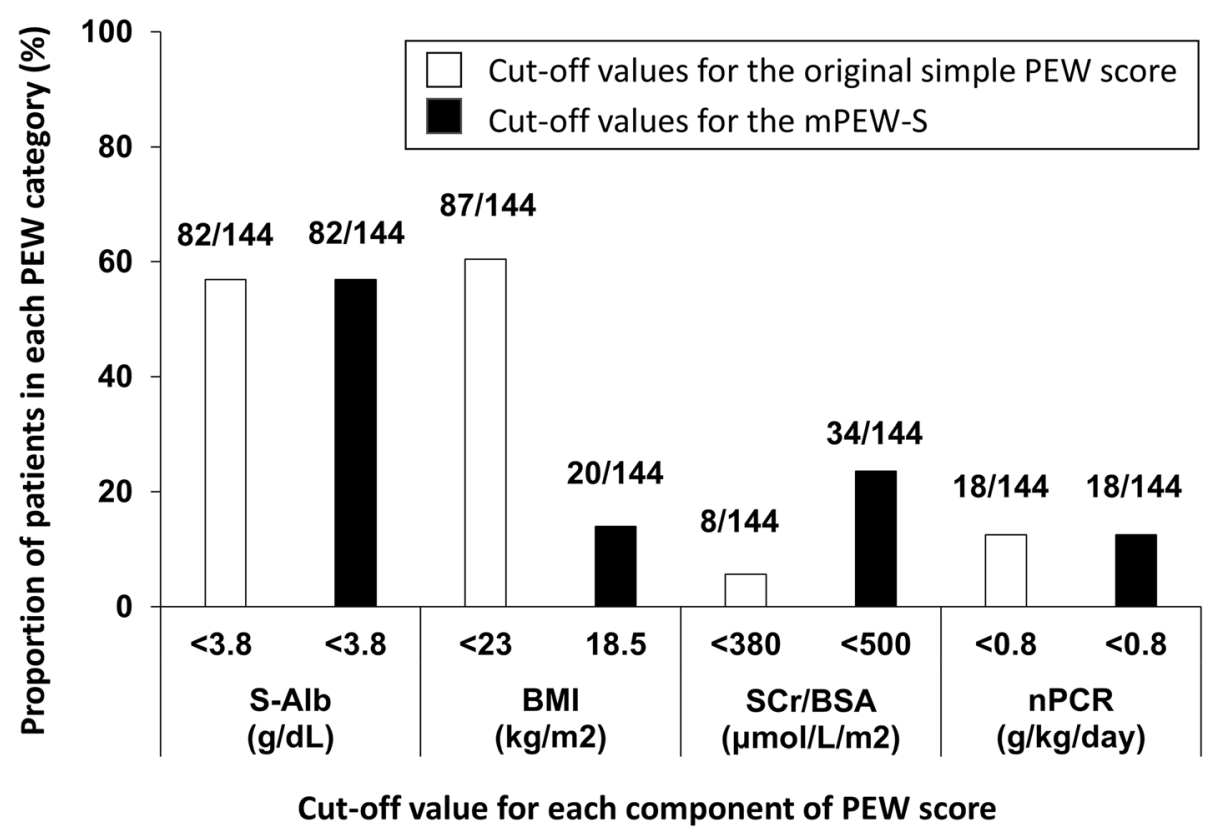

Fig. 1 Number of patients in each subcategory of PEW diagnostic criteria proposed by the ISRNM based on the original simple PEW score and mPEW-S $(n=144)$. BMI body mass index, BSA body surface area, ISRNM International Society of Renal Nutrition and Metabolism, $m P E W-S$ modified simple PEW score, $n P C R$ normalized protein catabolic rate, PEW protein-energy wasting, S-Alb serum albumin, S-Cr serum creatinine 
Table 2 Patient characteristics at baseline stratified by mPEW-S

\begin{tabular}{|c|c|c|c|c|c|}
\hline Risk group & Total & G1 & $\mathrm{G} 2$ & G3 & $P$ for \\
\hline mPEW-S & $0-4$ & 0,1 & 2 & 3,4 & \\
\hline Patients' number & $n=144$ & $n=60$ & $n=63$ & $n=21$ & \\
\hline \multicolumn{6}{|l|}{ Demographic data } \\
\hline Age, years & $62 \pm 12$ & $57 \pm 12$ & $63 \pm 11$ & $71 \pm 12$ & $<0.001$ \\
\hline Male sex, $n(\%)$ & $85(59)$ & $41(68)$ & $32(51)$ & $12(57)$ & 0.147 \\
\hline Diabetic nephropathy, $n$ (\%) & $17(11.9)$ & $7(12)$ & $6(10)$ & $4(19)$ & 0.554 \\
\hline History of cardiovascular diseases, $n(\%)$ & $20(14)$ & $4(7)$ & $12(19)$ & $4(19)$ & 0.062 \\
\hline Dialysis history, years & $11(5-22)$ & $14(8-27)$ & $7(3-19)$ & $8(7-14)$ & 0.228 \\
\hline $\mathrm{Kt} / \mathrm{V}$ for urea & $1.71 \pm 0.31$ & $1.68 \pm 0.33$ & $1.75 \pm 0.27$ & $1.66 \pm 0.34$ & 0.858 \\
\hline Normalized protein catabolic rate, $\mathrm{g} / \mathrm{kg} /$ day & $0.93 \pm 0.17$ & $0.96 \pm 0.15$ & $0.95 \pm 0.15$ & $0.78 \pm 0.18$ & $<0.001$ \\
\hline Dry weight, kg & $57.0 \pm 10.9$ & $59.7 \pm 10.5$ & $56.8 \pm 10.4$ & $49.8 \pm 10.7$ & $<0.001$ \\
\hline Body mass index, $\mathrm{kg} / \mathrm{m}^{2}$ & $22.2 \pm 3.5$ & $22.7 \pm 3.6$ & $22.5 \pm 3,1$ & $19.8 \pm 3.7$ & 0.006 \\
\hline \multicolumn{6}{|l|}{ Blood and serum biochemical parameters } \\
\hline Hemoglobin, g/dL & $11.2 \pm 1.1$ & $11.4 \pm 0.9$ & $11.0 \pm 1.3$ & $10.9 \pm 1.0$ & 0.023 \\
\hline Albumin, $\mathrm{g} / \mathrm{dL}$ & $3.7 \pm 0.3$ & $4.0 \pm 0.1$ & $3.6 \pm 0.3$ & $3.4 \pm 0.2$ & $<0.001$ \\
\hline C-reactive protein, $\mathrm{mg} / \mathrm{dL}$ & $0.1(0.1-0.3)$ & $0.1(0.1-0.2)$ & $0.2(0.1-0.4)$ & $0.4(0.1-1.9)$ & 0.004 \\
\hline Urea nitrogen, mg/dL & $64 \pm 14$ & $67 \pm 12$ & $65 \pm 13$ & $51 \pm 15$ & $<0.001$ \\
\hline Creatinine, $\mathrm{mg} / \mathrm{dL}$ & $10.7 \pm 2.5$ & $11.8 \pm 2.2$ & $10.7 \pm 2.3$ & $7.8 \pm 1.6$ & $<0.001$ \\
\hline Calcium, mg/dL & $9.4 \pm 0.5$ & $9.6 \pm 0.5$ & $9.2 \pm 0.5$ & $9.1 \pm 1.0$ & $<0.001$ \\
\hline Phosphate, mg/dL & $4.6 \pm 0.9$ & $4.8 \pm 0.8$ & $4.5 \pm 0.9$ & $4.6 \pm 1.0$ & 0.114 \\
\hline PTH (intact assay), pg/mL & $72(41-117)$ & $82(45-123)$ & $62(36-112)$ & $76(49-90)$ & 0.161 \\
\hline \multicolumn{6}{|l|}{ Medications } \\
\hline Anti-hypertensive drugs, $n$ (\%) & $90(54)$ & $38(63)$ & $40(63)$ & $12(57)$ & 0.689 \\
\hline ESAs, n (\%) & $122(85)$ & $50(83)$ & $54(86)$ & $18(86)$ & 0.730 \\
\hline VDRAs, $n(\%)$ & $106(74)$ & $44(73)$ & $47(75)$ & $15(71)$ & 0.937 \\
\hline Phosphate binders, $n$ (\%) & $115(80)$ & $48(80)$ & $52(83)$ & $15(71)$ & 0.970 \\
\hline Cinacalcet hydrochloride, $n$ (\%) & $36(25)$ & $14(23)$ & $14(22)$ & $8(38)$ & 0.322 \\
\hline
\end{tabular}

Data are mean $\pm S D$, median (interquartile range), or number (percentage)

ESAs erythropoiesis stimulating agents, $G$ subgroups divided by the mPEW-S, Kt/V plasma volume (V) cleared of urea (Kt) during hemodialysis relative to the distribution volume of urea, mPEW-S modified simple protein-energy wasting score, PTH parathyroid hormone, VDRAs vitamin D receptor activators

\section{Kaplan-Meier curves for all-cause death in the three groups divided by the MPEW-S}

During the median observation period of 5.7 years, 37 patients died of any-cause. Numbers of death were 6 in G1, 19 in G2, and 12 in G3. Figure 3 shows non-adjusted Kaplan-Meier curves for all-cause death according to subgroups stratified by the mPEW-S. Patients in G2 and G3 showed a higher incidence rate of all-cause death compared with those in G1 (log-rank test, $P<0.05$ ).

\section{Association between the MPEW-S and the risk for all-cause} mortality examined by Cox proportional hazards model

To further determine the association between the mPEW-S and all-cause death, we estimated the hazard risk for allcause death in each subgroup by applying the Cox proportional hazards risk model. As shown in Table 3, unadjusted and age- and sex-adjusted hazard ratios for all-cause death in G3 were significantly higher than those in G1. The association remained significant even after adjustment for several confounding factors: HR $(95 \% \mathrm{CI})$ for $\mathrm{G} 2,3.10$ (1.16-8.26); and for G3, 5.68 (1.85-17.45), respectively. The multivariable-adjusted $\mathrm{HR}(95 \% \mathrm{CI})$ for every 1 score increase in the mPEW-S was 1.67 (1.16-2.40).

\section{Comparison of the c-statistic of the ROC curves, NRI, and} IDI

Finally, to compare the predictability performance for all-cause mortality between the original simple PEW score and our mPEW-S, we compared the c-statistics, NRI, and IDI by setting the original simple PEW score as reference. As shown in Fig. 4, the c-statistic of the original simple PEW score was 0.582 , whereas that of mPEW-S was 0.695 , and the difference was statistically significant $(P=0.016)$. The cutoff value of $\mathrm{mPEW}-\mathrm{S}$ for 


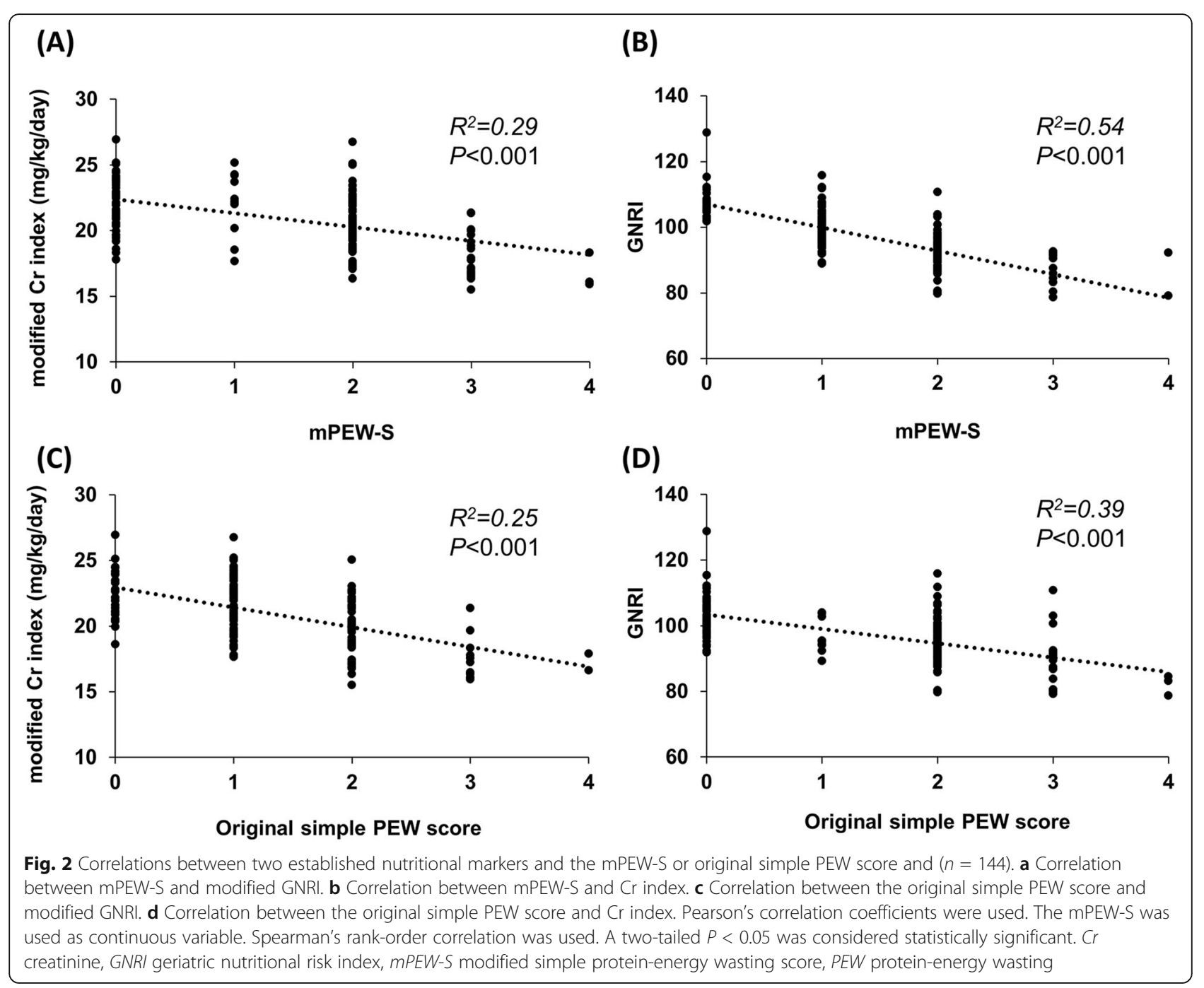

all-cause mortality was 2 , where sensitivity was $84 \%$ and specificity was $50 \%$. The cutoff value of the original simple PEW score for all-cause mortality was 2, the sensitivity was $60 \%$, and the specificity was $65 \%$. Furthermore, both NRI and IDI were significantly different between the original PEW score and MPEW-S: NRI, $-0.667(-0.975,-0.359), P<0.001$; IDI, -0.077 $(-0.116,-0.039), P<0.001$, respectively.

\section{Discussion}

In the present study, we proposed the MPEW-S, which was modified from the original simple PEW score consisting of serum albumin and Cr levels, BMI, and nPCR, by adjusting the cutoff values of those parameters suitable for Japanese patients receiving hemodialysis. The mPEW-S for these patients was significantly and negatively associated with the GNRI and modified $\mathrm{Cr}$ index, previously established good nutrition indexes [11, 12]. A higher mPEW-S was significantly associated with an increased risk for all-cause mortality, even after adjustment for several confounding factors. Furthermore, when predictability performance for all-cause mortality was compared, mPEW-S was superior to the original simple PEW score. Our results suggest that the mPEW-S can be a useful tool to stratify nutritional status and death risk and identify candidate patients for nutritional intervention among Japanese patients receiving hemodialysis. In addition, our study indirectly confirmed that the four nutritional parameters used in the semiquantitative assessment of PEW are valid in combination and useful for the evaluation of nutritional status of these patients.

Diagnosis of PEW is a challenging theme in patients receiving hemodialysis. Because there has been no single diagnostic marker or tool to perfectly determine whether a patient is PEW or not, clinical studies focusing on PEW inevitably require diagnostic definition of PEW by combining one or more of the nutrition-related surrogates to allocate patients into a binary variable pertaining to PEW. A variety of nutritional surrogates have been proposed for the evaluation of nutritional status in 


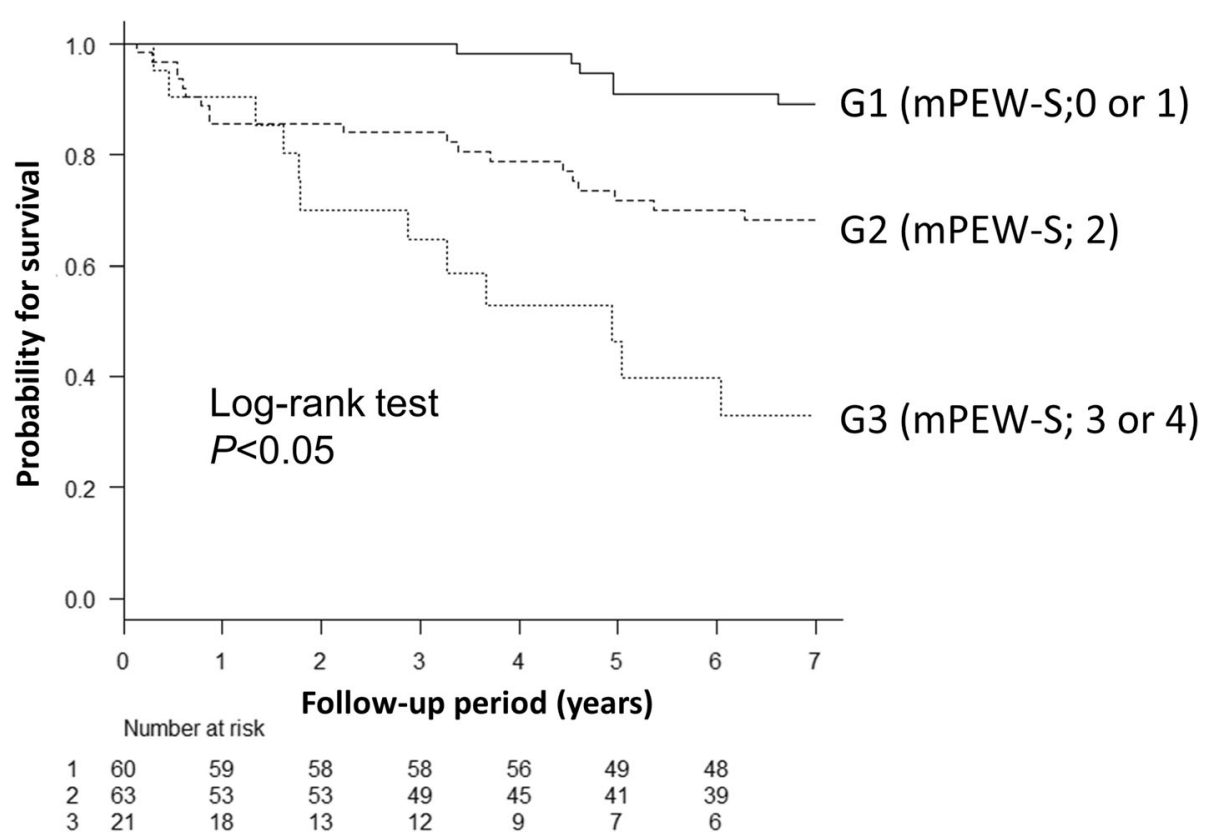

Fig. 3 Non-adjusted Kaplan-Meier curves in the three groups divided by the mPEW-S ( $n=144)$. A log-rank test was used to compare survival curves among groups. A two-tailed $P<0.05$ was considered statistically significant. $\mathrm{MPEW}$-S modified simple protein-energy wasting score. G1, patients whose MPEW-S was 0 or 1; G2, patients whose mPEW-S was 2; G3, patients whose mPEW-S was 3 or 4

hemodialysis patients [3, 6-9, 15-17]. Among the potential surrogates, serum levels of albumin and $\mathrm{Cr}$, BMI, and nPCR were chosen for PEW assessment. Each of the four surrogates corresponds to the component of the PEW subcategory proposed by the ISRNM and is also an established surrogate when used as a single marker. Notably, the Dialysis Outcomes and Practice Patterns Study showed that combination of two nutritional markers such as serum levels of albumin, Cr, and CRP and BMI are better than single nutritional marker [18]. These observations are acceptable because each marker provides only partial information on nutritional status. The combination of multiple surrogates enables us to assess nutritional status in a multifaceted way and offers a better prediction than a single surrogate. Indeed, the
Malnutrition-Inflammation Score is another representative and gold standard tool using a multifaceted assessment of nutritional status [3]. The assessment of malnutrition-inflammation-atherosclerosis syndrome, however, contains a subjective element and interobserver differences are occasionally problematic; this assessment requires experienced staff and is unsuitable for a local dialysis center. In contrast, the MPEW-S may be useful at the level of the local dialysis clinic as a simple and objective tool for periodic nutritional assessment.

The GNRI and $\mathrm{Cr}$ index are established nutritional indexes often used for the evaluation of nutritional status in patients receiving hemodialysis [11, 12]. Increasing evidence has shown that those two indexes are good predictors for the development of cardiovascular events

Table 3 Hazard ratios for the incidence of all-cause death in each subgroup stratified by mPEW-S $(n=144)$

\begin{tabular}{|c|c|c|c|c|c|c|c|c|c|c|}
\hline \multirow{2}{*}{$\begin{array}{l}\text { Groups stratified by } \\
\text { mPEW-S }\end{array}$} & \multirow{2}{*}{$\begin{array}{l}\text { No of events/ } \\
\text { No of patients }\end{array}$} & \multicolumn{3}{|l|}{ Unadjusted } & \multicolumn{3}{|c|}{ Age- and sex-adjusted } & \multicolumn{3}{|c|}{ Multivariable- adjusted } \\
\hline & & HR $(95 \%$ Cl) & $P$ value & $\begin{array}{l}P \text { for } \\
\text { trend }\end{array}$ & $\mathrm{HR}(95 \% \mathrm{Cl})$ & $P$ value & $\begin{array}{l}P \text { for } \\
\text { trend }\end{array}$ & $\mathrm{HR}(95 \% \mathrm{Cl})$ & $P$ value & $\begin{array}{l}P \text { for } \\
\text { trend }\end{array}$ \\
\hline $\mathrm{G} 1(0,1)$ & $6 / 60$ & 1 (reference) & & & 1 (reference) & & & 1 (reference) & & \\
\hline G2 (2) & $19 / 63$ & $3.63(1.45-9.10)$ & 0.006 & $<0.001$ & $3.25(1.28-8.24)$ & 0.013 & 0.001 & $3.10(1.16-8.26)$ & 0.024 & 0.005 \\
\hline G3 $(3,4)$ & $12 / 21$ & $9.60(3.58-25.77)$ & $<0.001$ & & $5.31(1.84-15.36)$ & 0.002 & & $5.68(1.85-17.45)$ & 0.002 & \\
\hline $\begin{array}{l}\text { Every } 1 \text { score } \\
\text { increase in } \mathrm{mPEW} \text {-S }\end{array}$ & & $2.02(1.47-2.85)$ & $<0.001$ & & $1.60(1.17-2.27)$ & 0.006 & & $1.67(1.16-2.40)$ & $<0.001$ & \\
\hline
\end{tabular}

Unadjusted and multivariable-adjusted HRs were analyzed by the Cox proportional hazard model. The multivariable-adjusted model included age, sex, diabetic nephropathy, history of cardiovascular events, dialysis history, blood hemoglobin, serum levels of log CRP, albumin-corrected calcium, phosphate, and parathyroid hormones. A two-tailed $P$ value $<0.05$ was considered statistically significant

$\mathrm{Cl}$ confidence interval, $C R P C$-reactive protein, $H R$ hazard ratio, $G$ subgroups divided by the $\mathrm{mPEW}-\mathrm{S}, \mathrm{mPEW}$ - $S$ modified simple protein-energy wasting score 


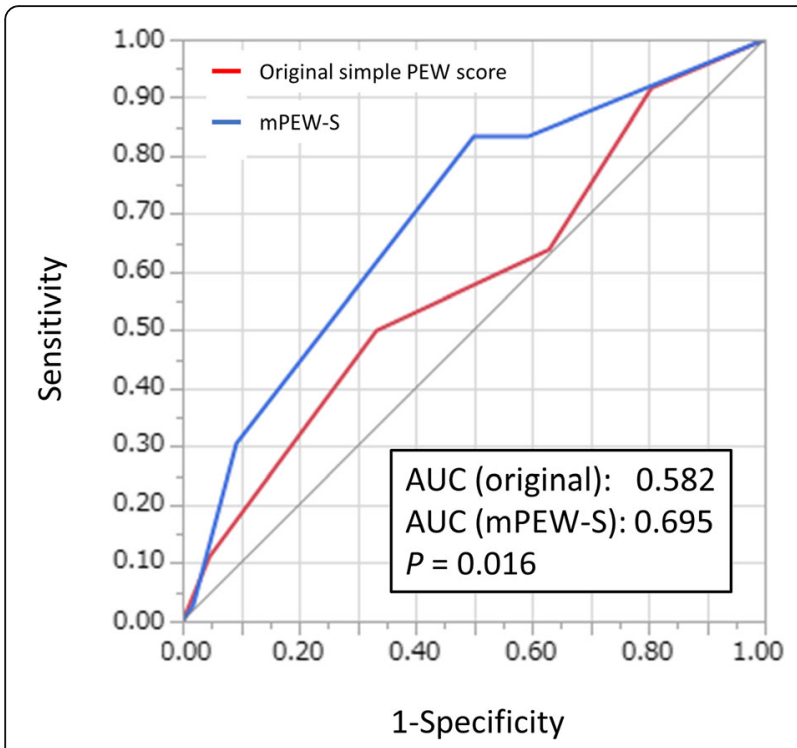

Fig. 4 Comparison of ROC curves between the original PEW score and mPEW-S $(n=144)$. A two-tailed $P<0.05$ was considered statistically significant. MPEW-S modified original PEW score, PEW protein-energy wasting, $R O C$ receiver operating characteristics

and bone fracture, as well as infection-related and all-cause death in patients undergoing hemodialysis $[11,12,19-21]$. The GNRI is correlated with the Malnutrition-Inflammation Score, which is now regarded as the gold standard for diagnosing PEW or malnutrition [22]. The modified $\mathrm{Cr}$ index is a marker of skeletal muscle mass and is also correlated with GNRI [23]. In the present study, our mPEW-S was significantly associated with both indexes. These observations suggest that our MPEW-S is a useful nutritional maker in the prediction of mortality in the hemodialysis population.

In the present study, mPEW-S was superior to the original simple PEW score. This is because the original cutoff values of the nutritional status were made for European patients and not for the Japanese. In other words, the cutoff values for each nutritional surrogate should be adjusted depending on the racial, ethnic, and social backgrounds. In this regard, our mPEW-S score was adjusted for Japanese patients receiving hemodialysis and can be used as a good surrogate for nutritional status and predictor of all-cause mortality in that population.

We are aware that our study has several limitations. First, our mPEW-S was derived from data obtained at a single Japanese hemodialysis center and comprised a relatively small sample size. Accordingly, the cutoff values of each $\mathrm{mPEW}-\mathrm{S}$ parameter should be validated by a larger independent hemodialysis population such as using nationwide database of hemodialysis patients held by the Japanese Society for Dialysis Therapy. Second, because the number of death events in each mPEW-S group was very small, the patients were divided into three subgroups when Kaplan-Meier curves and hazard risk models were applied and the risk stratification in each mPEW-S group was not accomplished. Accordingly, other subgroupings based on the MPEW-S might have altered the observed association in the present study. Third, although our mPEW-S was closely associated with pre-established nutritional indexes such as the GNRI and $\mathrm{Cr}$ index, the cutoff values were mainly based on the dataset of our 144 Japanese hemodialysis patients and rounded after considering the distribution of those values based on the current study and database of Japanese hemodialysis patients provided by The Japanese Society for Dialysis Therapy (https://www.jsdt.or.jp/ Overview_2.html). Hence, our mPEW-S should be validated with other Japanese hemodialysis populations. Fourth, because of the small sample size and outcome number, we included several confounding factors in the multi-variable adjustment. Hence, given that the known and unknown confounding factors were not adjusted, the association between the MPEW-S and all-cause death may be altered. Fifth, it is unclear whether our mPEW-S is a good predictor of other clinical outcomes such as cardiovascular disease events, bone fracture, or hospitalization. Therefore, further studies are necessary to determine the usefulness and validity of the mPEW-S developed in our study.

\section{Conclusions}

Our data showed that the MPEW-S for Japanese patients receiving maintenance hemodialysis was well correlated with pre-established nutritional risk indexes and associated with an increased risk of all-cause mortality. The mPEW-S can be a useful screening tool to detect malnutrition and identify candidates for nutritional support, especially in Japanese patients. Further studies are necessary to determine the external validity of the MPEW-S as a nutritional marker and whether the mPEW-S can be a useful tool for the prediction of other clinical outcomes in patients receiving maintenance hemodialysis. However, the mPEW-S should be cautiously used until it is validated by a larger hemodialysis population.

\section{Supplementary information}

Supplementary information accompanies this paper at https://doi.org/10. 1186/s41100-020-00289-6.

Additional file 1.

\section{Abbreviations}

BMI: Body mass index; BSA: Body surface area; Cl: Confidence interval; Cr: Creatinine; CKD: Chronic kidney disease; GNRI: Geriatric nutritional risk index; HR: Hazard ratio; IRI: Integrated reclassification improvement; ISRN M: International Society of Renal Nutrition and Metabolism; mPEW-

S: Modified PEW score; NRI: Net reclassification improvement; PEW: Protein 
energy wasting; PTH: Parathyroid hormone; ROC: Receiver operating characteristics

\section{Acknowledgements}

We are grateful to Andrea Baird, M.D., from Edanz Group (https://en-authorservices.edanzgroup.com/) for editing a draft of this manuscript.

\section{Authors' contributions}

Conception and study design: SY, MTo, and MTa; data acquisition: SY, ST, AH, SS, MTo, MTa, and KT; data analysis interpretation: SY, MTo, MTa, HY, HA, TN; statistical analysis: SY and TN; draft writing: SY; revision of the paper: SY, TN, ST, AH, SS, MTo, MTa, HO, HH, KT, and TK; supervision or mentorship: $\mathrm{HO}, \mathrm{HH}$, $\mathrm{TN}, \mathrm{KT}$, and TK. Each author contributed important intellectual content during manuscript drafting and accepts accountability for the overall work by ensuring that questions pertaining to the accuracy or integrity of any portion of the work are appropriately investigated and resolved. Each author has approved the submitted version of the paper. TK takes responsibility that this study has been reported honestly, accurately, and transparently; that no important aspects of the study have been omitted; and that any discrepancies from the study as planned have been explained.

\section{Funding}

None.

\section{Availability of data and materials}

The datasets generated during and/or analyzed during the current study are available from the corresponding author on reasonable request.

\section{Ethics approval and consent to participate}

The study was conducted in accordance with the guidelines of the Declaration of Helsinki and approved by the Institutional Review Board at Fukuoka Dental College (approval number: 2012-256).

\section{Consent for publication}

Not applicable.

\section{Competing interests}

None.

\section{Author details}

${ }^{1}$ Department of Medicine and Clinical Science, Graduate School of Medical Sciences, Kyushu University, 3-1-1 Maidashi, Higashi-Ku, Fukuoka 8128582, Japan. ${ }^{2}$ Fukuoka Renal Clinic, 4-6-20 Watanabe-dori, Chuo-Ku, Fukuoka 8100004, Japan. ${ }^{3}$ Department of Medicine, Fukuoka Dental College, 2-15-1 Tamura, Sawara-Ku, Fukuoka 8140193, Japan. ${ }^{4}$ Department of Nephrology, Nara Medical University, 840 Shijo-Cho, Kashihara, Nara 6348521, Japan.

\section{Received: 7 March 2020 Accepted: 18 August 2020}

Published online: 28 August 2020

\section{References}

1. Zha Y, Qian Q. Protein nutrition and malnutrition in CKD and ESRD. Nutrients. 2017;9(3):208.

2. Koppe L, Fouque D, Kalantar-Zadeh K. Kidney cachexia or protein-energy wasting in chronic kidney disease: facts and numbers. J Cachexia Sarcopenia Muscle. 2019;10(3):479-84

3. Kalantar-Zadeh $\mathrm{K}$, Kopple JD, Block G, Humphreys MH. A malnutritioninflammation score is correlated with morbidity and mortality in maintenance hemodialysis patients. Am J Kidney Dis. 2001;38(6):1251-63.

4. Fouque D, Kalantar-Zadeh K, Kopple J, Cano N, Chauveau P, Cuppari L, Franch H, Guarnieri G, Ikizler TA, Kaysen G, Lindholm B, Massy Z, Mitch W, Pineda E, Stenvinkel P, Treviño-Becerra A, Wanner C. A proposed nomenclature and diagnostic criteria for protein-energy wasting in acute and chronic kidney disease. Kidney Int. 2008;73(4):391-8.

5. Moreau-Gaudry X, Jean G, Genet L, Lataillade D, Legrand E, Kuentz F, Fouque D. A simple protein-energy wasting score predicts survival in maintenance hemodialysis patients. J Ren Nutr. 2014;24(4):395-400.

6. Iseki K, Kawazoe N, Fukiyama K. Serum albumin is a strong predictor of death in chronic dialysis patients. Kidney Int. 1993;44(1):115-9.

7. Kovesdy CP, Kalantar-Zadeh K. Accuracy and limitations of the diagnosis of malnutrition in dialysis patients. Semin Dial. 2012;25(4):423-7.
8. Kalantar-Zadeh K, Supasyndh O, Lehn RS, McAllister CJ, Kopple JD Normalized protein nitrogen appearance is correlated with hospitalization and mortality in hemodialysis patients with $\mathrm{Kt} / \mathrm{V}$ greater than 1.20. J Ren Nutr. 2003;13(1):15-25.

9. Patel SS, Molnar MZ, Tayek JA, Ix JH, Noori N, Benner D, Heymsfield S, Kopple JD, Kovesdy CP, Kalantar-Zadeh K. Serum creatinine as a marker of muscle mass in chronic kidney disease: results of a cross-sectional study and review of literature. J Cachexia Sarcopenia Muscle. 2013;4(1):19-29.

10. Lopes AA, Tong L, Thumma J, Li Y, Fuller DS, Morgenstern H, Bommer J, Kerr PG, Tentori F, Akiba T, Gillespie BW, Robinson BM, Port FK, Pisoni $\mathrm{RL}$. Phosphate binder use and mortality among hemodialysis patients in the Dialysis Outcomes and Practice Patterns Study (DOPPS): evaluation of possible confounding by nutritional status. Am J Kidney Dis. 2012; 60(1):90-101.

11. Yamada K, Furuya R, Takita T, Maruyama Y, Yamaguchi Y, Ohkawa S, Kumagai $\mathrm{H}$. Simplified nutritional screening tools for patients on maintenance hemodialysis. Am J Clin Nutr. 2008;87(1):106-13.

12. Canaud B, Granger Vallée A, Molinari N, Chenine L, Leray-Moragues H, Rodriguez A, Chalabi L, Morena M, Cristol JP. Creatinine index as a surrogate of lean body mass derived from urea Kt $N$, pre-dialysis serum levels and anthropometric characteristics of haemodialysis patients. PLoS One. 2014; 9(3):e93286.

13. Payne RB, Little AJ, Williams RB, Milner JR. Interpretation of serum calcium in patients with abnormal serum proteins. Br Med J. 1973;4(5893):643-66.

14. Kazama JJ. Japanese Society of Dialysis Therapy treatment guidelines for secondary hyperparathyroidism. Ther Apher Dial. 2007;11(Suppl 1):S44-7.

15. Herselman M, Esau N, Kruger JM, Labadarios D, Moosa MR. Relationship between serum protein and mortality in adults on long-term hemodialysis: exhaustive review and meta-analysis. Nutrition. 2010;26(1):10-32.

16. Eriguchi R, Obi Y, Streja E, Tortorici AR, Rhee CM, Soohoo M, Kim T, Kovesdy CP, Kalantar-Zadeh K. Longitudinal associations among renal urea clearancecorrected normalized protein catabolic rate, serum albumin, and mortality in patients on hemodialysis. Clin J Am Soc Nephrol. 2017;12(7):1109-17.

17. Stenvinkel P, Gillespie IA, Tunks J, Addison J, Kronenberg F, Drueke TB, Marcelli D, Schernthaner G, Eckardt KU, Floege J, Froissart M, Anker SD, ARO Steering Committee. Inflammation modifies the paradoxical association between body mass index and mortality in hemodialysis patients. J Am Soc Nephrol. 2016;27(5):1479-86.

18. Lopes AA, Bragg-Gresham JL, Elder SJ, Ginsberg N, Goodkin DA, Pifer T, Lameire N, Marshall MR, Asano Y, Akizawa T, Pisoni RL, Young EW, Port FK. Independent and joint associations of nutritional status indicators with mortality risk among chronic hemodialysis patients in the Dialysis Outcomes and Practice Patterns Study (DOPPS). J Ren Nutr. 2010;20(4):224-34.

19. Matsukuma Y, Tanaka S, Taniguchi M, Nakano T, Masutani K, Hirakata H, Kitazono T, Tsuruya K. Association of geriatric nutritional risk index with infection-related mortality in patients undergoing hemodialysis: The QCohort Study. Clin Nutr. 2019;38(1):279-87.

20. Yamada S, Taniguchi M, Tokumoto M, Yoshitomi R, Yoshida H, Tatsumoto N Hirakata H, Fujimi S, Kitazono T, Tsuruya K. Modified creatinine index and the risk of bone fracture in patients undergoing hemodialysis: The Q-Cohort Study. Am J Kidney Dis. 2017;70(2):270-80.

21. Arase H, Yamada S, Yotsueda R, Taniguchi M, Yoshida H, Tokumoto M, Nakano T, Tsuruya K, Kitazono T. Modified creatinine index and risk for cardiovascular events and all-cause mortality in patients undergoing hemodialysis: The Q-Cohort study. Atherosclerosis. 2018;275:115-23.

22. Szeto CC, Kwan BC, Chow KM, Law MC, Li PK. Geriatric nutritional risk index as a screening tool for malnutrition in patients on chronic peritoneal dialysis. J Ren Nutr. 2010;20(1):29-37.

23. Hwang W, Cho MS, Oh JE, Lee JH, Jeong JC, Shin GT, Kim H, Park I. Comparison of creatinine index and geriatric nutritional risk index for nutritional evaluation of patients with hemodialysis. Hemodial Int. 2018; 22(4):507-14.

\section{Publisher's Note}

Springer Nature remains neutral with regard to jurisdictional claims in published maps and institutional affiliations. 